\title{
Exploiting triatomine behaviour: alternative perspectives for their control
}

\author{
Claudio R Lazzari ${ }^{1 /+}$, Marcelo G Lorenzo² \\ ${ }^{1}$ Institut de Recherche sur la Biologie de I'Insecte, UMR 6035 CNRS, Université François Rabelais, Av. Monge, Parc Grandmont, 37200 \\ Tours, France ${ }^{2}$ Instituto René Rachou-Fiocruz, Belo Horizonte, MG, Brasil
}

Living in close association with a vertebrate host and feeding on its blood requires different types of adaptations, including behavioural adjustements. Triatomines exhibit particular traits associated with the exploitation of their habitat and food sources and these traits have been the subject of intense analysis. Many aspects of triatomine behaviour have been relatively well characterised and some attempts to exploit the behaviours have been undertaken. Baited traps based on host-associated cues, artificial refuges and light-traps are some of the tools used. Here we discuss how our knowledge of the biology of Chagas disease vectors may help us sample and detect these insects and even increase the efficiency of control measures.

Key words: Chagas disease - vector control - behavioural traits

The diverse lifestyles of the main Chagas disease vector species and the different habitats they live in require multiple tools and specific strategies for interrupting the vectorial transmission of Trypanosoma cruzi. Chemical interventions to control triatomine populations are important, but the surveillance of low density infestations inside or near human housings should play a role as well. Among the multiple approaches successfully employed for the control of these insect pests, those based on behavioural traits have not been thoroughly exploited when dealing with triatomines.

Before we consider different possibilities for exploiting the behaviour of Chagas disease vectors, we need to briefly summarise their main behavioural traits, particularly those that are susceptible to being targeted and used for population control. Detailed information on particular aspects of triatomine behaviour can be found in the review article on the chemical ecology of Chagas disease vectors by Cruz-López et al. (2001) and information on the way triatomines obtain and make use of information to find blood sources can be found in the review by Guerenstein and Lazzari (2009).

\section{A brief account of triatomine behaviour}

Triatomines spend most of their lives hidden inside refuges, but they leave them when their hosts are resting. Inside human houses and in the nests and burrows of forest-dwelling vertebrates, triatomines are more active at night, with two periods of maximum activity: one just

Financial support: Agence Nationale de la Recherche, Centre National de la Recherche Scientifique, Université François Rabelais

+ Corresponding author: claudio.lazzari@univ-tours.fr

Received 25 March 2009

Accepted 26 May 2009 after dusk and another at dawn (Lazzari 1992). These two periods are devoted to finding different resources. The first burst of activity is to seek out a host and the second is to find a refuge to spend the day (Lorenzo \& Lazzari 1996, 1998). Other activities, such as mating, oviposition and dispersion, are also performed during one or both of these time periods. Once inside a refuge, they fall into an inactive state, or akinesis, promoted by signals such as assembling pheromones and physical contact with the substrate, as well as by internal circadian clocks. They also "wake up" due to signals from an internal clock to start a new activity cycle (Guerenstein \& Lazzari 2009).

When searching for food, triatomines rely on multimodal cues that include physical and chemical signals emitted by warm-blooded vertebrates and the air currents that transport those signals. The presence of a potential host can be revealed by odours that stimulate these insects. This chemical stimulation can trigger an anemotactic response, i.e., the ability to follow air currents that guide them to a host over a long range (Taneja \& Guerin 1995, 1997, Guerenstein \& Guerin 2001, Barrozo et al. 2003, Barrozo \& Lazzari 2004a, b, 2006). When the insect is within a few meters, the heat emitted by the host, particularly infrared radiation, is detected. This increases the amount of information available to the insects not only for spatial localisation, but also for the recognition of the host (Wigglesworth \& Gillett 1934, Lazzari \& Núñez 1989, Flores \& Lazzari 1996, Barrozo et al. 2003). Triatomine bugs are able to distinguish the temperature of different objects and orient themselves towards those that have a temperature characteristic of endothermic hosts (Lazzari \& Núñez 1989).

As far as we know, triatomine communication makes use of two different sensory channels: a chemical channel and a vibrational channel. Chemical communication is involved in many aspects of their lives and pheromones promoting assembling, mating and alarm behaviours have been partially characterised (Baldwin et al. 1971, Schofield \& Patterson 1977, Lorenzo Figueiras et 
al. 1994, Manrique \& Lazzari 1995, Lorenzo Figueiras \& Lazzari 1998a, b, Cruz-López et al. 2001, Vitta et al. 2002, 2007, Manrique et al. 2006, Pontes et al. 2008). Vibrational communication associated with stridulation has been described in male-female interactions related to a female's rejection of copulation attempts by males. Stridulation also occurs as a response to mechanical perturbation (Manrique \& Lazzari 1994, Roces \& Manrique 1996, Manrique \& Schilman 2000, Schilman et al. 2001, Lazzari et al. 2006).

Another important biological trait of triatomines is the ability of adults to disperse over long-ranges at night (Lehane \& Schofield 1977, 1981, Schofield et al. 1991, 1992, Vazquez-Prokopek et al. 2004, Gurevitz et al. 2006). Flying triatomines are attracted to artificial lights, including those of houses (Schofield \& Matthews 1985, Minoli \& Lazzari 2006, Carbajal de la Fuente et al. 2007). Undoubtedly, this is a key factor leading to their colonisation of anthropic environments.

Some of the methods currently used to monitor and control Chagas disease vectors can be seen as means of exploiting the aforementioned behaviours. For instance, government programs promoting the improvement of houses aim to reduce the number of potential refuges available for insects, forcing them to remain exposed to predators and pesticides. This can hinder the establishment of domiciliary colonies. Triatomines need to find dark and narrow places to rest in close contact with the substrate during the day. In the same sense, GomezNúñez and Maria boxes act as artificial refuges, providing protection, physical contact and darkness (GomezNúñez 1965, Wisnivesky-Colli et al. 1988).

Live host-baited traps used for sampling triatomines in sylvatic habitats are another example of techniques that take advantage of triatomine behaviour. These traps exploit the host searching behaviour of the insects by attracting them (Noireau et al. 1999, 2002). Other devices have been proposed that use baker's yeast as a source of carbon dioxide and other volatile substances (Guerenstein et al. 1995, Lorenzo et al. 1998, 1999, Pires et al. 2000, Pimenta et al. 2007).

Other biological characteristics of triatomines can be exploited to control their populations or to detect their presence in houses as well as in their natural habitats. We will discuss some of them as well as the possibility of putting them into practice, but first we should mention some general principles of Triatomine behaviour.

\section{Using insect behavioural traits}

Taking advantage of a pest's behaviour for control purposes has revealed itself to be a successful, even efficient, strategy in many cases. Examples of its effectiveness in controlling different species of insects are abundant in the literature. Insects of various orders (i.e., Lepidoptera, Diptera), with different lifestyles (i.e., culture pests, haematophagous flies) living in different environments (i.e., cultures, open savannah areas, forests, buildings) have been the target of different behavioural control strategies (Shorey \& McKelvey 1977, Greenblatt \& Lewis 1983).

\section{Exploiting triatomine activity}

Triatomines acting as vectors for Chagas disease are essentially nocturnal. Even though the activity patterns for all relevant species have not been characterised, the major vectors, Triatoma infestans and Rhodnius prolixus, have a bimodal pattern of activity (Constantinou 1979, Lazzari 1992). Triatomines leave their refuges at dusk to search for food and to find refuges for the next day (Lorenzo \& Lazzari 1998). During this period of time, it is thought that they stay away from both hosts and refuges, probably performing their diuresis in a protected place away the shelter. It has also been shown that these insects do most of their feeding and are most sensitive to some host signals in the early night hours (Barrozo et al. 2004, Bodin et al. 2008).

Because of their activity patterns, triatomines are not only more exposed and active at night, but they are also more vulnerable to mechanical and chemical attacks. Their vulnerability to chemical attacks is due to the distension of their abdominal cuticle (Ianowski et al. 1998), which becomes more fragile and permeable to pesticides just after feeding (Fontan \& Zerba 1992). Preliminary experiments performed recently in T. infestans also showed that their sensitivity to insecticides is much higher at night (I Amelotti et al., unpublished observations).

This evidence suggests that chemical control measures may be more efficient when they are applied at night because this assures that the triatomines will come into direct contact with active chemicals. Even though insecticides may remain active for long periods of time, it is possible for these bugs to avoid treated surfaces. It is obvious that spraying at night may not always be possible for practical reasons. This is especially true when an infestation calls for the intervention of qualified technical teams. However, when spraying is performed by local people living in infested houses, the application of insecticides or the manual search for triatomines in the night hours is recommended.

\section{Exploiting triatomine communication}

A great deal of work has been done concerning triatomine communication. The triatomine vibratory channel hardly seems exploitable for pest control and its use for detecting the presence of hidden bugs in walls and roofs by detecting their vibrations has not yet been investigated. In any case, its application could be very limited given that it would require sophisticated techniques and expensive equipment to be a feasible control method.

The use of pheromones for attracting insects or disturbing their communication during sensitive periods of their life, for example their mating period, has been used as a tool for controlling different insect pests. Several instances where pheromones are used in triatomine communication have been identified. These instances are related to mating, alarm, assembling and substratemarking behaviours. Among them, the aggregation signals present in triatomine faeces appear to be the most promising for triatomine control for several reasons: (i) faeces samples can be easily obtained from rearing facilities, since the paper or cardboard sheets usually used 
as substrates are a rich source for triatomine waste; (ii) modern chemical methods should be able to unravel the composition of these pheromones and eventually produce synthetic versions of them; (iii) the pheromones remain effective for almost two weeks (Lorenzo Figueiras \& Lazzari 2000); (iv) the way triatomines convey information using the pheromones found in faeces is understood (Lorenzo \& Lazzari 1996); (v) pheromones in faeces are able to attract and assemble triatomines in an unspecific fashion (Lorenzo Figueiras \& Lazzari 1998a, 2002, Pires et al. 2002, Vitta et al. 2007); (vi) pheromones in faeces are used in the context of finding shelters (Lorenzo \& Lazzari 1996).

Artificial refuges baited with the chemicals composing the aggregation signal appear to be a control solution that deserves attention. Current box sensors are able to detect the presence of wandering bugs, but their efficiency could be improved by associating them with a lure that actively recruits triatomines. The chemical composition of this signal appears to be common to several species of triatomines, and synthetic mixtures of these compounds have been shown to be capable of luring triatomines into artificial shelters (MG Lorenzo, unpublished observations).

\section{Exploiting triatomine habitat selection}

As indicated above, the chemical environment associated with a refuge (i.e., the presence of aggregation signals) is important for inducing aggregation in triatomines. In addition, the physical properties of the refuge are also known to play a role in shelter selection. Refuges offering intense physical contact due to their intricate surfaces appear to be the most appropriate. The microclimate inside the refuge also seems to affect the selection of a place to rest (Lorenzo \& Lazzari 1999). Nevertheless, controlling temperature or relative humidity inside an artificial shelter does not seem feasible for a low-cost field device. Adequate materials that tend to reduce humidity may be a suitable alternative. Finally, dark surfaces have been proven to be preferred by inactive triatomines and this should be taken into account when designing artificial refuges (Reisenman et al. 2000).

\section{Exploiting triatomine dispersion}

Triatomines are able to undertake relatively long dispersive flights and the attraction of triatomines to artificial light sources has also been widely reported. From an epidemiological point of view, attraction to light plays an important role in the invasive process of triatomines into human dwellings. What causes these photophobic insects to be attracted to artificial lights remained puzzling until recently? New evidence suggests that a change in the sign of the phototactic response to punctual lights takes place after the imaginal moult (CR Lazzari et al., unpublished observations). This means that triatomines do not invade houses by chance or due to navigational errors as would be expected in the case of amenotactic error. On the contrary, triatomines are actively attracted by incandescent lamps (Minoli \& Lazzari 2006). This means that every dispersing individual, not just a fraction of them, could actively approach lights and eventually settle in houses.
Sampling insects using light traps has been a common practice for many years. Several studies have been done to determine the types of light source that are most appropriate for these practices. Results to date have revealed that white light bulbs and even portable light sources (battery or fuel operated) are quite efficient for this purpose (Carbajal de la Fuente et al. 2007). More work is needed to analyse whether their efficiency could be improved by adjusting the light wavelength of the lamps or adding spectral filters to them.

\section{Exploiting triatomine host-seeking}

Triatomines, like many other blood-sucking insects, are attracted by different physical and chemical hostassociated cues. Heat, water vapour and odours released by vertebrate hosts allow triatomines to detect their presence, localise them in space and orient to their position. In addition to the cues emitted by hosts, air currents play two major roles: they disperse these signals and offer triatomines directional cues about the source of stimuli (odour-modulated anemotaxis).

Our knowledge about the effects of vertebrate cues on triatomine behaviour as well as on the detection thresholds makes these cues useful for capturing triatomines. Two different approaches have been tested to date. The first one uses a live host as bait in a relatively simple capture device known by triatomine field researchers as a Noireau's trap. These simple devices are efficient for capturing sylvatic triatomines in natural ecotopes. One reason these traps are so efficient is that they use the "ideal bait", a real host. Vertebrates are sources of a complex mixture of stimuli that include signals from different sensory modalities that attract triatomines. Even though imitating the performance of a real host is difficult, for practical and ethical reasons it would be helpful to find alternative baits to replace live hosts. Attempts have been made to use baker's yeast as a lure since it is a cheap and easy to handle source of carbon dioxide and other host volatiles. Although some successful tests have been conducted in laboratory conditions, field tests are still necessary to assess their efficiency. A drawback to using yeast cultures as baits is that they are short-lived and, being liquids, are difficult to use in vertical or narrow places.

Other baits and traps have been proposed, but they remain to be tested under rigorous laboratory and field conditions. Almost none of them, however, exploit the response of triatomines to warm objects, probably because it is difficult to incorporate a durable and inexpensive source of heat into a trap.

In conclusion, in addition to intervention measures such as improving the walls of rural houses and adjusting the time of insecticide spraying, two types of devices seem to be promising for the control, surveillance and sampling of triatomines. These devices are based on triatomine shelter utilisation and host-seeking behaviour. Judging by the utility of sensor boxes and Noireau's traps, these alternatives seem to be quite promising. Nevertheless, the chance of success for developing novel and efficient capture or detection devices can be increased if some basic principles are taken into account: (i) we will probably never be able to develop bait that is more at- 
tractive than a real living host. Therefore, the best option would be to imitate a host as closely as possible to attract triatomines; (ii) given the first principle, any artificial bait will be more efficient in the absence of real hosts; (iii) triatomines are eclectic and opportunistic in their feeding behaviour. We should look for general attractants such as heat, $\mathrm{CO}_{2}$, or fatty acids, rather than for specific cues associated with particular host species; (iv) triatomines, as other haematophagous insects, make use of multimodal cues to locate hosts. Thus, combinations of physical and chemical stimuli, even if more difficult to standardise, will have a better chance of attracting these bugs; (v) triatomines make parsimonious use of information. This means that the same odour may have different interpretations depending on the context in which it is presented. For instance, isobutiric acid is the main component of the alarm signals of several species and is also released by hosts (Guerenstein \& Guerin 2001, Manrique et al. 2006). Depending on its concentration and the presence of other volatile compounds, triatomines can be attracted or repelled by its presence; (vi) a deep knowledge of the biology of triatomines is indispensable, especially the behavioural and temporal context of potential signals. Without this knowledge, we risk using supposedly attractive cues in inappropriate situations; (vii) in order to have a real significance for triatomines, attractants need to be presented in precise combinations and not simply thrown together. The statement "the more attracting cues we associate with a device, the better it will work" is simply not true.

Triatomine excrement finely illustrates how behavioural manipulation traps should be conceived. Even though the attractiveness of triatomine faeces has been well established (Schofield \& Patterson 1977, Lorenzo Figueiras et al. 1994), associating them with pitfall traps that require that the insects to fall inside proved to be ineffective (Guerenstein et al. 1995). A close inspection of triatomine behaviour revealed that they were effectively attracted to trap surroundings by their faeces. Nevertheless, faecal odours were unable to induce triatomines to fall into traps as they usually did when the trap was baited with host-related signals (Guerenstein et al. 1995). This is quite logical given that we are proposing that specific insect behaviours, i.e., falling after odours, are usually associated with host search (when wandering triatomines let themselves fall on a host from ceilings). However, this behaviour cannot be triggered in the context of refuge search. They do not enter shelters by jumping inside. Thus, the behavioural context associated with each particular signal must be known and taken into account to increase the chances of success. Otherwise, our designs will be meaningless and the chances of success will be significantly reduced.

\section{REFERENCES}

Baldwin WF, Knight AG, Lynn KR 1971. A sex pheromone in the insect Rhodnius prolixus (Hemiptera: Reduviidae). Can Entomol 103: $18-22$.

Barrozo RB, Lazzari CR 2004a. The response of the blood-sucking bug Triatoma infestans to carbon dioxide and other host odours. Chem Senses 294: 319-329.
Barrozo RB, Lazzari CR 2004b. Orientation behaviour of the bloodsucking bug Triatoma infestans to short-chain fatty acids: synergistic effect of L-lactic acid and carbon dioxide. Chem Senses 29: 833-841.

Barrozo RB, Lazzari CR 2006. Orientation response of haematophagous bugs to $\mathrm{CO}_{2}$ : the effect of the temporal structure of the stimulus. J Comp Physiol A 192: 827-831.

Barrozo RB, Manrique G, Lazzari CR 2003. The role of water vapour in the orientation behaviour of the blood-sucking bug Triatoma infestans Hemiptera: Reduviidae. J Insect Physiol 494: 315-321.

Barrozo RB, Minoli SA, Lazzari CR 2004. Circadian rhythm of behavioural responsiveness to carbon dioxide in the blood-sucking bug Triatoma infestans Heteroptera: Reduviidae. J Insect Physiol 50: $249-254$.

Bodin A, Barrozo RB, Couton L, Lazzari CR 2008. Temporal modulation and adaptive control of the behavioural response to odours in Rhodnius prolixus. J Insect Physiol 549: 1343-1348.

Carbajal de la Fuente A, Minoli SA, Lopes CM, Noireau F, Lazzari CR, Lorenzo MG 2007. Flight dispersal of the Chagas disease vectors Triatoma brasiliensis and Triatoma pseudomaculata in North-Eastern Brazil. Acta Trop 101: 115-119.

Constantinou C 1979. Circadian rhythms in insects, PhD Thesis, University of London, $270 \mathrm{pp}$.

Cruz López L, Malo EA, Rojas JC, Morgan ED 2001. Chemical ecology of triatomine bugs: vectors of Chagas disease. Med Vet Entomol 15: 351-357.

Flores GB, Lazzari CR 1996. The role of the antennae in Triatoma infestans: orientation towards thermal sources. J Insect Physiol 42: 344-440.

Fontán A, Zerba E 1992. Influence of the nutritional state of Triatoma infestans over the insecticidal activity of DDT. Comp Bioch Physiol C 101: 589-591.

Gómez-Núñez JC 1965. Desarrollo de un nuevo metodo para evaluar la infestacion intradomiciliaria por Rhodnius prolixus. Acta Cient Venez 16: 26-31.

Greenblatt JA, Lewis WJ 1983. Chemical environment manipulation for pest insects control. Environl Manag 7: 35-41.

Guerenstein PG, Guerin PM 2001. Olfactory and behavioural responses of the blood-sucking bug Triatoma infestans to odours of vertebrate hosts. $J$ Exp Biol 204: 585-597.

Guerenstein PG, Lazzari CR 2009. Host-seeking: how triatomines acquire and make use of information to find blood. Acta Trop, in press.

Guerenstein PG, Lorenzo MG, Núñez JA, Lazzari, CR 1995. Baker's yeast an attractant for baiting traps for Chagas' disease vectors. Experientia 518: 834-837.

Gurevitz JM, Ceballos LA, Kitron U, Gürtler RE 2006. Flight initiation of Triatoma infestans (Hemiptera: Reduviidae) under natural climatic conditions. J Med Entomol 43: 143-150.

Ianowski JP, Manrique G, Núñez JA, Lazzari CR 1998. Feeding is not necessary for triggering plasticization of the abdominal cuticle in haematophagous bugs. J Insect Physiol 445: 379-384.

Lazzari CR 1992. Circadian organization of locomotion activity in the haematophagous bug Triatoma infestans. J Insect Physiol 38: 895-903.

Lazzari CR, Manrique G, Schilman P 2006. Vibrational communication in Triatominae (Heteroptera: Reduviidae). In Claridge \& 
Drosopoulos (eds.), Insect sounds and communication: physiology, behaviour, ecology and evolution, CRC Press, USA, UK, 552 pp.

Lazzari CR, Núñez JA 1989. The response to radiant heat and the estimation of the temperature of distant sources in Triatoma infestans. J Insect Physiol 35: 525-529.

Lehane MJ, Schofield CJ 1977. Preliminary report on flight by some triatomine bugs. Trans R Soc Trop Med Hyg 70: 526.

Lehane MJ, Schofield CJ 1981. Field experiments of dispersive flight by Triatoma infestans. Trans R Soc Trop Med Hyg 75: 399-400.

Lorenzo Figueiras AN, Kenigsten A, Lazzari CR 1994. Aggregation in the haematophagous bug Triatoma infestans: chemical signals and temporal pattern. J Insect Physiol 40: 311-316.

Lorenzo Figueiras AN, Lazzari CR 1998a. Aggregation behaviour and interspecific responses in three species of triatomine. Mem Inst Oswaldo Cruz 93: 133-137.

Lorenzo Figueiras AN, Lazzari CR 1998b. Aggregation in the haematophagous bug Triatoma infestans: a novel assembling factor. Physiol Entomol 23: 33-37.

Lorenzo Figueiras AN, Lazzari CR 2000. Temporal changes in the aggregation response in Triatoma infestans. Mem Inst Oswaldo Cruz 95: 889-892.

Lorenzo Figueiras AN, Lazzari CR 2002. Aggregation behaviour and interspecific responses in Rhodnius prolixus Stål. Mem Inst Oswaldo Cruz 97: 569-571.

Lorenzo MG, Lazzari CR 1996. The spatial pattern of defecation in Triatoma infestans and the role of faeces as a chemical mark of the refuge. J Insect Physiol 42: 903-907.

Lorenzo MG, Lazzari CR 1998. Activity pattern in relation to refuge exploitation and feeding in Triatoma infestans (Hemiptera: Reduviidae). Acta Trop 70: 163-170.

Lorenzo MG, Lazzari CR 1999. Temperature and relative humidity affect the selection for refuges in Triatoma infestans, vector of Chagas disease. Acta Trop 72: 241-249.

Lorenzo MG, Manrique G, Pires HHR, de Brito Sanchez MG, Diotaiuti L, Lazzari CR 1999. Yeast cultures volatiles as attractants for Rhodnius prolixus. Electroantenogram responses and capture in yeast-baited traps. Acta Trop 72: 119-124.

Lorenzo MG, Reisenman CE, Lazzari CR 1998. Triatoma infestans can be captured under natural climatic conditions using yeastbaited traps. Acta Trop 70: 277-284.

Manrique G, Lazzari CR 1994. Sexual behaviour and stridulation during mating in Triatoma infestans (Hemiptera: Reduviidae). Mem Inst Oswaldo Cruz 89: 629-633.

Manrique G, Lazzari CR 1995. Existence of a sex pheromone in Triatoma infestans (Hemiptera: Reduviidae): I. Behavioural evidence. Mem Inst Oswaldo Cruz 90: 645-648.

Manrique G, Schilman PE 2000. Two different vibratory signals in Rhodnius prolixus (Hemiptera: Reduviidae). Acta Trop 77: 271-278.

Manrique G, Vitta ACR, Ferreira RA, Zani CL, Unelius CR, Lazzari CR, Diotaiuti L, Lorenzo MG 2006. Chemical communication in Chagas disease vectors. Source, identity and potential function of volatiles released by the metasternal and brindley's glands of Triatoma infestans adults. J Chem Ecol 32: 2035-2052.

Minoli SA, Lazzari CR 2006. Take-off activity and orientation of triatomines (Heteroptera: Reduviidae) in relation to the presence of artificial lights. Acta Trop 97: 324-330.
Noireau F, Abad-Franch F, Valente SA, Dias-Lima A, Lopes CM, Cunha V, Valente VC, Palomeque FS, de Carvalho-Pinto CJ, Sherlock I, Aguilar M, Steindel M, Grisard EC, Jurberg J 2002. Trapping Triatominae in silvatic habitats. Mem Inst Oswaldo Cruz 97: 61-63.

Noireau F, Flores R, Vargas F 1999. Trapping sylvatic Triatominae (Reduviidae) in hollow trees. Trans R Soc Trop Med Hyg 93: 13-14.

Pimenta FE, Diotaiuti L, Lima ACL, Lorenzo MG 2007. Evaluation of cultures of Saccharomyces cerevisae as baits for Triatoma dimidiata and Triatoma pallidipennis. Mem Inst Oswaldo Cruz 102: 229-231.

Pires HHR, Diotaiuti L, Lazzari CR, Lorenzo MG 2000. Performance of yeast-baited traps with Triatoma sordida, Triatoma brasiliensis, Triatoma pseudomaculata and Panstrongylus megistus in laboratory assays. Rev Panam Salud Publica 7: 384-388.

Pires HHR, Lorenzo MG, Diotaiuti L, Lazzari CR, Lorenzo Figueiras AN 2002. Aggregation behaviour in Panstrongylus megistus (Burmeister, 1835): intra and interspecific responses. Acta Trop 81: 47-52.

Pontes GB, Bohman B, Unelius CR, Lorenzo MG 2008. Metasternal gland volatiles and sexual communication in the triatomine bug, Rhodnius prolixus. J Chem Ecol 34: 450-457.

Reisenman CE, Lorenzo Figueiras AN, Giurfa M, Lazzari CR 2000. Interaction of visual and olfactory cues in the aggregation behaviour of the haematophagous bug Triatoma infestans. J Comp Physiol A 186: 961-968.

Roces F, Manrique G 1996. Different stridulatory vibrations during sexual behaviour and disturbance in the blood-sucking bug Triatoma infestans (Hemiptera: Reduviidae). J Insect Physiol 42: $231-238$

Schilman PE, Lazzari CR, Manrique G 2001. Comparison of disturbance stridulations in five species of triatominae bugs. Acta Trop 79: $171-178$.

Schofield CJ, Lehane MJ, McEwan P, Catala SS, Gorla DE 1991. Dispersive flight by Triatoma sordida. Trans $R$ Soc Trop Med Hyg 85: 676-678.

Schofield CJ, Lehane MJ, McEwen P, Catala SS, Gorla DE 1992. Dispersive flight by Triatoma infestans under natural climatic conditions in Argentina. Med Vet Entomol 6: 51-56.

Schofield CJ, Matthews JN 1985. Theoretical approach to active dispersal and colonization of houses by Triatoma infestans. $J$ Trop Med Hyg 88: 211-222.

Schofield CJ, Patterson JW 1977. Assembly pheromone of Triatoma infestans and Rhodnius prolixus nymphs (Hemiptera: Reduviidae). J Med Entomol 13: 727-734.

Shorey HH, McKelvey JJ 1977. Chemical control of insect behaviour, John Wiley \& Sons, New York, 512 pp.

Taneja J, Guerin PM 1995. Oriented responses of triatomines bugs Rhodnius prolixus and Triatoma infestans to vertebrate odours on a servosphere. J Comp Physiol A 176: 455-464.

Taneja J, Guerin PM 1997. Ammonia attracts the haematophagous bug Triatoma infestans: behavioural and neurophysiological data on nymphs. J Comp Physiol A 181: 21-34.

Vazquez-Prokopec GM, Ceballos LA, Kitron U, Gurtler RE 2004. Active dispersal of natural populations of Triatoma infestans (Hemiptera: Reduviidae) in rural Northwestern Argentina. $J$ Med Entomol 41: 614-621. 
Vitta ACR, Lorenzo Figueiras AN, Lazzari CR, Diotaiuti L, Lorenzo MG 2002. Aggregation mediated by faeces and footprints in Triatoma pseudomaculata (Heteroptera: Reduviidae), a Chagas disease vector. Mem Inst Oswaldo Cruz 97: 865-867.

Vitta ACR, Mota TRP, Diotaiuti L, Lorenzo MG 2007. The use of aggregation signals by Triatoma brasiliensis (Heteroptera: Reduviidae). Acta Trop 101: 147-152.
Wigglesworth VB, Gillett JD 1934. The function of the antennae in Rhodnius prolixus (Hemiptera) and the mechanism of orientation to the host. J Exp Biol 11: 120-139.

Wisnivesky-Colli C, Paulone I, Chuit R, Perez A, Segura EL 1988. A new method for the detection of reinfested households during surveillance activities of control programmes of Chagas' disease. Rev Arg Microbiol 20 (Suppl. 1): 96-102. 